\title{
6. Charting a framework for regulatory stewardship
}

\section{INTRODUCTION}

In the previous chapter, I examined the ways in which certain actors in health research-particularly RECs_-are affected by regulation, and similarly, the ways in which they can affect regulation. The findings revealed that research ethics review is an essential component of health research regulation and the ethics review system overall appears to be operating relatively smoothly, at least in comparison to previous decades. At the same time, though, the evidence suggests that several regulatory components can be refined.

In this chapter, I unpack further the significance of the liminality of RECs and the ability of actors within the health research regulatory space to serve as 'regulatory stewards'. I do so by taking up the normative dimension of anthropology of regulation, suggesting a model of what a regulatory framework for health research oversight ought to look like if it were to incorporate the findings from this empirical investigation. This would include explicit endorsement of regulatory stewardship and a charting of how protection and promotion can and should work together in regulatory design and practice.

This proposed framework has application at two levels, which can be seen as both top-down and bottom-up: (1) the government and managing regulators (e.g. Department of Health and Social Care, HRA), and (2) RECs and regulatees (e.g. researchers, sponsors, institutions). As the evidence in Chapter 5 indicates, RECs are embedded in multiple overlapping, interconnecting regulatory spaces, yet their roles and the roles of other actors are not always manifest in regulation. Further, the conversations between regulators, namely between RECs and the HRA, can be sporadic and at times less effective as compared to the conversations between regulators and regulatees (here, being RECs and researchers). This can cause disconnected spaces to appear within a given regulatory space where hazards may occur. A reformulated framework could work to improve regulatory conversations between actors, provide ongoing 
opportunities for 'regulatory play' to emerge, and shift the burden and emphasis away from procedural work and towards flexibility and experimentation in ethics review. What I suggest, in other words, is a refinement of the existing framework, not wholesale change. Nonetheless, this is a refinement that can be worth exploring to reveal the full range and weight of the impact of RECs within and throughout regulatory practices in health research regulation.

In what follows, first, I expand on the significance of the liminality of RECs and unpack the concept of regulatory stewardship. I argue that regulatory stewardship serves as a manifestation of liminality and deserves greater instantiation in regulation. I draw on extant examples within the UK's RESs that demonstrate how regulatory stewardship can play a vital role for researchers in navigating complex regulatory terrain. Then, I consider what should happen when a proposed research project involves a conflict of principles or values-in this context, the potential for protection versus promotion. I then conclude with a proposal for a more processual regulatory framework that enables regulatory stewards to assist in accommodating potential harms and maximizing research outcomes, and that creates a regulatory space within which there is more room for regulators to protect and promote, including room to experiment in working through these principles together with other actors.

\section{THE LIMINALITY OF RECs-REGULATORY STEWARDSHIP}

From what I observed over the course of a year, ethics review is less an administrative process, where ethical considerations of proposed research end once a favourable opinion is given by a REC, than it is a process of ongoing support, dialogue, and education. If we accept Bobbie Farsides's claim that ' $[t]$ he goal of an ethics committee is to facilitate ethically sound practice, and to encourage researchers to honour their moral responsibilities towards participants', ${ }^{1}$ we should further accept that this cannot be adequately accomplished within a regulatory framework that charges ethics committees to engage merely in regulatory verification of ethical standards, scientific value, and accordance with law.

Facilitation of ethically sound practice and inculcation of moral responsibilities in researchers necessitates a framework of regulatory

1 Calliope (Bobbie) Farsides, 'The Ethics of Clinical Research' in Sue Eckstein (ed), Manual for Research Ethics Committees (6th edn, CUP 2003) 13. 
stewardship (the prudent guidance of one or more actors across regulatory thresholds), whereby a range of actors, including RECs, work with researchers, sponsors, and others not just to achieve regulatory compliance, but also to work through stages in the research lifecycle, all the while instilling ethical norms of good scientific conduct. Thus, stewardship is a stand-alone regulatory role and collective responsibility that should be assumed by different actors at multiple stages. For their part, RECs should have an expanded role to play in the research lifecycle, but as I will discuss, they cannot and should not cover each and every stage. As regulatory stewardship permeates health research, all actors should view each other as crucial links in a chain that moves ethical research from design to approval to recruitment and action, and ultimately, to health improvement. With different actors embodying roles at different stages, connected by communicative channels that allow for a 'passing of the mantle', stewardship helps us think differently about what is going on in research and how each link connects to the other.

Chapter 5 illustrated in several ways how RECs are liminal actors. Relative to each other and to much of the public, RECs are black boxes, existing in multiple spaces, despite exhibiting a surprising degree of group homogeneity in approach and rituals. RECs engage in various mechanisms to evaluate research applications (e.g. balance, ranking, negotiation) that manifest themselves at a lower level of abstraction'good research design', 'competent investigator', 'favourable balance of harm and benefit', 'adequate informed consent'-which in turn yield 'ethical' judgements. Embodying a liberal approach that aims to eschew a paternalistic stance towards participants, RECs adopt a pragmatic ethics that is informed by members' intuition, feeling, and experience.

RECs do not fit the mould of a classic risk-based regulator. For example, we saw that they are also attuned to potential burdens as well as issues surrounding scientific design and law. Returning to the discussion first opened in Chapter 2, we saw that RECs' operative ethical deliberation is the facilitation of a context in which a fair choice is offered to participants whereby they can decide whether to participate in a project that presents ethically acceptable risks and burdens and is likely to answer, or at least contribute to, the research question it purports to address. Members adopt rituals in undertaking the process of ethics review that work best for them as individuals and as a committee. Through teamwork and consensus, they render an opinion that mediates the demands of science and society and achieves a kind of optimization of the similarly appreciated values of protection and promotion. The opinion allows a research protocol to transition, as colleagues and I have 
written elsewhere, 'from a mere proposition of involvement with participants to an actual plan of action with participants. This implicates a range of actors, and importantly, it further transforms individuals (be they healthy "volunteers" or patients) into active research participants'. ${ }^{2}$

Given the fluid jurisdiction between ethics, science, and law, and given their active role in steering behaviour, what kind of regulators are RECs? Can we accurately label them 'ethics committees'? As RECs become institutionalized and professionalized, acting as multi-faceted and multidisciplinary regulators of health research (concerned with, for example, minimizing risks and ensuring scientific and social value), and as more national and international regulations come into force that impact health research, RECs might be expected to act more as 'health research regulatory committees'. Indeed, the empirical research suggests that RECs are not mere ethics consultation groups. They do certainly engage in some form of ethics deliberation and discussion, but much more regulatory work is also being performed alongside other actors, including researchers. 'Health research regulatory committees' may well be a more accurate name to reflect what they do. ${ }^{3}$ And, if we do treat RECs more as health research regulatory committees, we would be well served to rethink their roles and the regulatory frameworks that govern them to better incorporate the regulatory processes they undertake.

What can we make of Deryck Beyleveld's claim that a 'dog cannot serve two masters, and the role of RECs, in fact, is solely to try to prevent unethical research. The facilitation of research is the role of other bodies'? ${ }^{4}$ Let us recall that liminality draws our attention to how actors experience and react to change, and that the evidence from Chapter 5 suggests that, if anything, recent changes in the law reflect alreadyexisting practices of RECs. RECs, managing regulators, and researchers share a common and desired goal of promoting research that is safe and of high quality. ${ }^{5}$ Actors in these regulatory spaces carry similar interests

2 Agomoni Ganguli-Mitra and others, 'Reconfiguring Social Value in Health Research Through the Lens of Liminality' (2016) 31 Bioethics 87, 89 (emphasis in original).

3 See also Sheelagh McGuinness, 'Research Ethics Committees: The Role of Ethics in a Regulatory Authority' (2008) 34 Journal of Medical Ethics 695 ('RECs act as regulatory authorities with concerns beyond those of ethical deliberation. I argue that RECs are regulatory rather than advisory').

4 Deryck Beyleveld, 'Law, Ethics and Research Ethics Committees' (2002) 21 Medicine and Law 57, 73.

5 Such a finding accords with Adam Hedgecoe's empirical research, which found that NHS RECs can proactively promote research. See Adam Hedgecoe, 
and shared responsibilities, helping each other to cross boundaries and deal with major moments of transition in the research lifecycle. My investigation has not indicated that RECs are guided by a single principle of participant protection. Research promotion is also very much present and at play-and welcomed-in their functions.

As we saw, regulators in this space can have a problematic relationship between each other, much more so than between regulators and regulatees. The HRA strives to chart a regulatory environment that enables researchers to bring a research project to light in a smooth and efficient manner; a critical component of this charting involves interactions with RECs. The relationship between the HRA and RECs can be politically fraught, though, drawing RECs into struggles for power with their managing regulatory authority. There is a strong desire by RECs, including REC Chairs and Managers, to preserve the sanctity of their black box and ethics of space. RECs simultaneously want more guidance from the HRA on regulatory developments (such as, during the time of my investigation, HRA Approval) and limited imposition on their everyday workings. That possible imposition of power is exemplified in the HRA's Ethical Review Form, which influences the processes of ethics review. The 'balance' managing regulators must achieve between sound coordination and overreaching diktat is a difficult one, particularly in a country with devolved administrations. The relatively limited communication channels with the HRA generally are viewed not as problematic per se; indeed, they may be beneficial. The HRA sees itself as providing a steering (i.e. catalysing), not controlling, role for RECs. For many REC members, that relatively light-touch approach is a value that reinforces the RECs' independence (or phrased somewhat differently, preserves their autonomy) and ability to reach decisions without fear of external pressure or loss of power over their domains of control.

More profoundly, the interactions between RECs and their managing regulators suggest that regulation is increasingly 'centred' where the state, through the Department of Health and Social Care, the HRA, and equivalent bodies in Wales, Northern Ireland, and Scotland, is exercising growing influence, but not necessarily control. There is also evidence, though, that the resources relevant to holding regulatory power and exercising capacities in research involving humans are dispersed. Never have the resources in this space been restricted to formal, state authority derived from legislation. Historically, and continuing through the present,

'Research Ethics Review and the Sociological Research Relationship' (2008) 42 Sociology 873. 
those resources have included expertise and organizational capacities shared between state and non-state bodies, including sponsors and funders.

RECs do serve to control access to the potentiality of research involving humans, but controlled access through their 'event licensing' system is buttressed by a facilitative ideology set within an 'ethics of space' - a conscious desire to promote research and, in turn, advance human health. And this, arguably, is the 'ethics' in the REC. Ethics is not about compliance or control, but rather about debate, reflection, values, argument, and justification. Legitimate and diverse disagreement can (and ought to) occur. As a matter of regulatory practice, then, an ethics of space must accommodate diversity, disagreement, and dissent across applications and across time. This in turn suggests that, by their nature, liminal regulatory spaces must be provided for RECs and applicants alike to explore and deliberate on the 'ethics'. Unsurprisingly, a substantial majority of REC members I interviewed and observed did not view protection and promotion as creating a conflict. Rather, their practice of working through both seems to instantiate the Declaration of Helsinki's Paragraph 23 recommendation to not only consider, comment on, and potentially approve a research protocol, but to offer 'guidance' on it as well. ${ }^{6}$

More questionable, though, is whether this practice instantiates Paragraph 8 of the Declaration of Helsinki and the GAfREC guidance, that is, that the goals of research and the researcher, while important, should always be secondary to the dignity, rights, and well-being of the research participant. Certainly, the dignity, rights, and well-being of research participants were always considered and respected in the REC meetings I observed, but it cannot be said that the interests of researchers and research were 'always' treated as 'secondary to the dignity, rights, safety and well-being of people taking part in research' ${ }^{7}$ Instead, the interests of researchers, research, and participants were often treated as aligned or even merged. Some REC members and regulators actively questioned the absolutist position taken in ethical guidelines that prioritize the individual over society. REC practices demonstrate that to protect is to promote. The blurring of the role hierarchy, or this long-standing ambiguity of role hierarchy in the UK if we consider the Royal College of Physicians Guidelines dating back to 1984, reflects, as with the fluidity of science

6 World Medical Association, Declaration of Helsinki (World Medical Association 2013) para 23.

7 GAfREC para 3.2.2. 
and ethics review, an incongruence between certain regulatory strategies and general practices that the HRA and other managing regulators may need to reassess.

Does this finding of regulatory connectivity impact the overall quality and effectiveness of health research regulation? Not in terms of REC practices, I would argue, but it does invite questions about the role RECs and other actors can play if provided more room to 'roam' throughout the regulatory space. RECs, I would argue, engage in a pragmatic form of instantiated regulation, translating written regulatory guidance from the HRA and other managing regulators into practical action that capitalizes on the relative interpretative flexibility of their regulatory texts. ${ }^{8}$ They enact situationally specific ways to implement the regulations (from the SOPs, GAfREC, and so on) that govern their practice in determining the ethical acceptability of research applications. And, their role as regulatory steward reflects a collectively negotiated, practical, doable solution that satisfies the spirit of the regulations. RECs indeed have more regulatory flexibility than first appears and part of this flexibility is based on 'interpersonal trust in instantiating and maintaining system trust' ${ }^{9}$ Even so, in this chapter I want to argue that more flexibility should be provided in the regulatory framework to enable specific actors to engage in this stewardship role and experiment with different ways of working through the stages of the research lifecycle. Liminality can help us to both recognize uncertainties that may arise across the research lifecycle, embrace them to a certain extent, potentially even exploit them, and pay attention to what is required to work through them.

I want to argue for regulatory stewardship's embeddedness in the regulatory framework because the empirical data suggest that RECs' knowledge control and gatekeeping activities have the potential to reach beyond the ex ante stage. The hybrid protectionist-promotionist model that operates in practice fosters an environment that both protects research participants and also facilitates responsible health research through proportionate regulation and coordinated alignment of ethics review and other regulatory processes. This can be operationalized not only at the early stage(s) of the research lifecycle, at the moment of research design and initial application, but also throughout the lifecycle in partnership with other regulatory actors where ongoing opportunities for 'regulatory play' can emerge. Crucial to this argument are the

8 Neil Stephens and others, 'Documenting the Doable and Doing the Documented: Bridging Strategies at the UK Stem Cell Bank' (2011) 41 Social Studies of Science 791.

9 ibid 808. 
research findings that suggest that the currently existing arm's-length approach from law is beneficial. By avoiding clearly defined roles of RECs and their procedural and substantive aspects, the law is actually helpful in promoting the normative behaviours that I recommend.

In what follows, I propose modifications to the existing regulatory framework by suggesting elements of regulatory stewardship that allow RECs to act as 'work groups' with their managing regulators, as well as regulatees. In so doing, I contend that if the 'regulatory conversations' 10 that RECs engage in with other actors are structured well (e.g. steps are enacted to avoid regulatory capture or inequity), one can mitigate the concerns about co-constructed 'organisational deviance' that Hedgecoe warned about in his discussion of the TGN1412 drug trial scandal at Northwick Park Hospital. ${ }^{11}$ Embedding regulatory stewardship, I contend, allows RECs to better engage with the processual and experiential dynamics of health research and instantiate a processual-oriented mode of regulation.

First, however, I propose a framework for working through protection and promotion, namely a deliberative and accommodating mode supported by a looping mechanism of transition for a research protocol that transforms it into something 'ethical' within a given moment of time and within particular spaces. Research passes through multiple liminal phases; ethicality is not guaranteed across each stage. As different actors and regulatory and ethical implications arise with each stage, RECs and others can play a crucial role in helping research and researchers follow these processes through each stage. Of course, stewardship is only as good as its weakest link in the chain. As different actors come into the fold across the research lifecycle, the mantle of stewardship through each of these stages must be passed smoothly and efficiently. Key to this is an effective regulatory design that enables robust and dynamic communication among all actors.

\section{WORKING THROUGH PROTECTION AND PROMOTION}

We have seen that REC members can utilize several different mechanisms to work through protection and promotion. As the bioethicist

\footnotetext{
10 See Julia Black, ‘Talking About Regulation’ [1998] Public Law 77.

11 Adam Hedgecoe, 'A Deviation from Standard Design? Clinical Trials, Research Ethics Committees, and the Regulatory Co-Construction of Organizational Deviance' (2014) 44 Social Studies of Science 59.
} 
Michael Dunn writes, 'aligning normative justification with policy and practice in research ethics is likely to require the introduction of novel governance frameworks that support an ethics committee's adjudication between general principles upon which people can reasonably disagree'. ${ }^{12}$ In the context of this book, the absence of an expressed theory of how the objectives of protection and promotion should be achieved necessitates the crafting of at least the outline of one.

The physician Simon Whitney argues that there are 'two major moral considerations in research with human subjects' that ethics committees must 'balance': the rights and welfare of research subjects and the 'shared interest in better treatments for disease'. ${ }^{13}$ My concern with this argument is that Whitney assumes that 'balance' is the correct (or best) operative mechanism to adequately reconcile the objectives of participant protection and research promotion. 'Balance', I argue, is both an ambiguous metaphor (for a scale of measurement) that is cognitively suspect in health research and also a mechanism that wrongly antagonizes the values at stake.

There is no mechanism within 'balance' that enables one to weigh competing claims. As the communication studies scholars Robert Patterson and Ronald Lee write: "On the one hand, "balance" evokes the precision of the objective scale; on the other, it evokes the democratic value of equity. As a result, "balance" connotes a process that is simultaneously precise and fair.' ${ }^{14}$ If there is no agency of balance, balance becomes a rhetorical construction of fairness and (pseudo-)objectivity. At most, one can trust that individuals and groups intersubjectively reach an acceptable balance between protection and promotion, whereby acceptability reflects a range of ethical acceptability. Moreover, the empirical research suggests that protection and promotion are not seen as oppositional values. 'Balance' would fail to capture the iterative, communicative, and fluid nature of ethical deliberations that seek to have protection and promotion work together. In sum, balance may not be the correct (or best) mechanism. The values of protection and promotion are unsuitable for a utilitarian calculus that positions them as oppositional. And, to the extent this 'balance' currently happens, it may

12 Michael Dunn, 'Getting the Justification for Research Ethics Review Right' (2013) 39 Journal of Medical Ethics 527, 528.

13 Simon Whitney, Balanced Ethics Review: A Guide for Institutional Review Board Members (Springer 2016) vii.

14 Robert Patterson and Ronald Lee, 'The Environmental Rhetoric of "Balance": A Case Study of Regulatory Discourse and the Colonization of the Public' (1997) 6 Technical Communication Quarterly 25, 35. 
well suffer from the same flaws or weaknesses as the risk-benefit calculus noted by several scholars. ${ }^{15}$

Thus, I advocate instead an iterative view of protection and promotion defined by process and tolerance, where both protection and promotion are generally treated simultaneously and relationally. Specifically, protection and promotion should be treated as twin objectives for regulators. The liminality of RECs suggests that there is a need for a deliberative space within which RECs can both negotiate the risks relevant to a research application and work with researchers to get to a point where the application can be deemed ethically acceptable. This deliberative space ought to be protected to capture and promote the fluid, processual nature of those deliberations. Tolerance indicates that, within this space, REC members should feel comfortable debating the strengths and weaknesses of a research project, and achieving some consensus position on how much risk they are willing to tolerate. This risk toleration, in turn, needs to be considered relative to the notion of research promotion. Thus, rather than viewing protection as a bright-line test, tolerance accommodates the fluid nature of ethics deliberation and the relative nature of risk, that is, a higher tolerance of greater risk if it is seen as reasonable in relation to the benefits to participants and society.

Moreover, I claim this approach should be iterative as RECs' regulatory roles should manifest themselves not only at the singular stage of ethics review, but also before and after in the research lifecycle. An ethically approved research project does not necessarily remain ethical throughout its duration. Both time and space can impact this judgement and liminality encourages actors to follow processes through their stages of transition. 'Ethical research', as determined by achievement of protection and promotion, must continually be created and re-evaluated as a research project progresses. Feedback loops (i.e. opportunities for, and various channels of, communication, dialogue, and negotiation) should be built into the regulatory framework to prevent a static and putatively binding approach to 'ethical research', thereby encouraging greater regulatory conversations that allow RECs to continually ensure research

15 See e.g. Annette Rid, 'Rethinking Risk-Benefit Evaluations in Biomedical Research' in Daniel Strech and Marcel Mertz (eds), Ethics and Governance of Biomedical Research (Springer 2016) 153; Michelle Meyer, 'Three Challenges for Risk-Based (Research) Regulation: Heterogeneity Among Regulated Activities, Regulator Bias, and Stakeholder Heterogeneity' in I Glenn Cohen and Holly Fernandez Lynch (eds), Human Subjects Research Regulation: Perspectives on the Future (MIT Press 2014). 
is ethical-which is to say, protecting participants and optimizing the research project's social and scientific value as it evolves.

Such an iterative view of protection and promotion would better recognize the liminal and thus processual enterprise of health research. It would also operationalize the language already contained in the Care Act 2014,16 the latest edition of the Royal College of Physicians Guidelines, ${ }^{17}$ and HRA guidance for REC members. ${ }^{18}$ Further, it would reinforce the Declaration of Helsinki's Paragraph 8: 'While the primary purpose of medical research is to generate new knowledge, this goal can never take precedence over the rights and interests of individual research subjects.' ${ }^{19}$ A couple of REC members expressed concern that this statement might imply the interests of science and society should not be considered in any assessment. As a REC Chair told me, 'you may attach less weight to them, but you need to attach some sort of weight to them' (P10). But if this assessment were treated as a weighing (i.e. balancing) of interests, undoubtedly they would always be weighed in favour of 'individual research subjects'. Thus, the problem is that balancing would fail to reflect the REC's role as not merely internally consultativedeliberations among themselves as to whether participants are adequately protected-but also as a promoter of ethical best practice that necessitates ongoing dialogue with other actors, foremost researchers themselves.

Such an approach to protection and promotion would work to avoid a 'bureaucratization of ethics', where research ethics is treated as equivalent to REC processes (and in particular, approval of consent forms and information sheets) and the scope of ethical concerns is narrowed to the front end of approvals of research proposals. Coupling this approach with regulatory stewardship allows for smoother navigation of spaces that emerge in-between actors and between stages in the research lifecycle.

16 Care Act 2014, s 110(2), stating that one of the HRA's objectives is 'to promote the interests of those participants and potential participants and the general public by facilitating the conduct of research that is safe and ethical' (emphasis added).

17 Royal College of Physicians, Guidelines on the Practice of Ethics Committees in Medical Research with Human Participants (4th edn, Royal College of Physicians 2007) 4 ('RECs have a duty to encourage important ethical research').

18 Health Research Authority, 'Information for Potential Research Ethics Service Committee Members' <www.hra.nhs.uk/documents/1025/standardapplication-pack-rec-members.pdf $>$ accessed 22 October 2019 ('The key duty of a REC is to protect the interests of research participants whilst at the same time facilitating ethical research').

19 Declaration of Helsinki (n 6) para 8. 
An iterative view allows RECs to escape the institutionally delineated time-space trap where their work is fixated on a specific moment in time and within a specific space of the committee meeting, and thereby possibly avoid a "permanent liminality-where uncertainties and antistructures prevail'.$^{20} \mathrm{RECs}$, along with other actors, may instead come to be seen as stewards that help guide health research through multiple thresholds: from research design, to ethics approval, to participant recruitment and consent, to data generation, to data analysis, to knowledge translation, and so on.

Having set out to offer a framework of working through protection and promotion that incorporates liminality as an analytic and normative frame, I now turn to suggest a normative model of what a regulatory framework for health research oversight ought to look like if it were to explicitly endorse regulatory stewardship and chart how protection and promotion can work together. As Chapter 5 explained, regulatory stewardship can be defined as the prudent guidance of one or more actors across regulatory thresholds-without which there is risk of failure, impairment, or harm - with a view to fulfilment of regulatory objectives and collective betterment. ${ }^{21}$ While stewardship is a somewhat well-known concept, ${ }^{22}$ regulatory stewardship is not. I argue that it can demonstrate considerable added value for all actors implicated in the network of health research ethics oversight in delivering and benefiting from efficient and effective navigation of regulatory landscapes. In so doing, I also chart the nature of regulatory stewardship's features and functions, and the different types of stewards that can exist to take on different functions.

20 Samuel Taylor-Alexander and others, 'Beyond Regulatory Compression: Confronting the Liminal Spaces of Health Research Regulation' (2016) 8 Law, Innovation and Technology 149, 174.

21 Adapted from Graeme Laurie and others, 'Charting Regulatory Stewardship in Health Research: Making the Invisible Visible?' (2018) 27 Cambridge Quarterly of Healthcare Ethics 333.

22 See e.g. World Health Organization, 'Stewardship' <www.who.int/health systems/stewardship/en/> accessed 22 October 2019; Lynn Jansen, 'Between Beneficence and Justice: The Ethics of Stewardship in Medicine' (2013) 38 Journal of Medicine \& Philosophy 50. 


\section{CHARTING A FRAMEWORK FOR REGULATORY STEWARDSHIP}

In what follows, I propose three elements (some with sub-parts) to improve the current regulatory framework for oversight of health research involving human participants. These elements flow naturally from the empirical results and as such should be charted. I begin with the element that imposes the least transaction cost and reflects most accurately what already occurs in practice, based on my research, and thus requires minimal regulatory change. I end with the element that may be more potentially disruptive to the current system and thus requires more extensive reform. The elements are proposed with a view towards a realistic, practical view of current resource constraints, both within the NHS and within RECs themselves. It is clear that RECs must be properly resourced to fulfil the roles expected of and practised by them. Moreover, regulatory administration must be in lockstep with research growth: to the extent the UK's research environment is in good health, so too must be the regulatory actors responsible for regulating research. The overall approach taken here is one that encourages greater cooperation among and integration of regulators and regulatees.

\subsection{Flexibility}

As this book has argued, RECs operate within a hybrid regulatory design: social control of research is divided between state-based actors (e.g. HRA, MHRA) and non-state actors (e.g. volunteer REC members, sponsors). Decision-making combines central and regional or local controls, and a multiplicity of actors are engaged in regulatory policymaking. Hybrid design is seen as fostering greater regulatory flexibility, but we have also seen that, within the health research regulatory space, RECs are increasingly tacking towards the state; a 'centring' regulation is occurring that may limit the potential for regulatory flexibility. RECs may feel curtailed in their ability to adapt ethical frameworks or standards to a given research project when faced with the threat of sanction from above. As I have argued, an ethics of space must accommodate diversity, disagreement, and dissent across applications and across time. Likewise, researchers may feel curtailed in their ability to adapt their research as it develops, still within reasonable ethical boundaries, out of fear of falling foul of an already-approved protocol. In both instances, a culture of caution and rigidity can come to dominate decision-making. RECs and researchers therefore should be enabled to 
decide and act on matters within a range of reasonableness. Not only will this allow the flourishing of sound and ethical health research grounded in conscience rather than compliance, it will allow RECs and researchers to adapt regulatory responses to changing environments (both within a specific research project and across types of research).

For example, REC SOPs have served to greatly improve clarity and consistency in structure and processes, but positivistic rule-following is not the only value at stake in ethics review; 'responsible conduct often runs obliquely to compliance with rules'. ${ }^{23}$ The length of REC SOPs have become colossal (a document now running to over 300 pages), and one wonders if something - flexibility and an opportunity to innovate-is getting lost in the drive to conform to such numerous standards. Through issuance of guidance with best practices, RECs should be encouraged to act with greater discretion to enable them to develop more innovative, experimental, and strategic approaches to their reviews. To this end, Annette Rid has called for a comprehensive and detailed ethical framework for risk-benefit evaluations centred on social and scientific value. ${ }^{24}$ I support this call, provided, however, that such a detailed ethical framework allows RECs some interpretive flexibility in how they undertake such evaluations. A rigid application of a framework, especially one that is comprehensive, may well lead to pushback or failure. As another example, managing regulators such as the HRA should be wary of prescribing procedural requirements that restrict what RECs can accomplish in conducting reviews both within and outwith scheduled monthly full committee meetings (such as rushing to get through six research ethics applications in three hours). Checklists should be treated as aide-memoires, not rigid forms to judge REC performance. As the bioethicist Linus Johnsson and colleagues write: 'If ethical guidelines are to actually inspire researchers to make better decisions, they must have a sufficiently high level of abstraction to give room for deliberation. They must never be allowed to degenerate into checklists.' 25

Perhaps the best example of enhanced regulatory flexibility, though, is greater tolerance for an ethics of space that encourages deliberation and debate regarding protection and promotion.

23 Linus Johnsson and others, 'Making Researchers Moral: Why Trustworthiness Requires More Than Ethics Guidelines and Review' (2014) 10 Research Ethics 29, 40.

24 Rid (n 15).

25 Johnsson and others (n 23) 42. 


\subsubsection{An ethics of space}

The empirical research suggests that REC members treat participant protection and research promotion as intertwined values that manifest themselves through the process of their review and in the course of their deliberations at REC meetings. In some cases it may be possible for RECs to focus first on protection and only thereafter on promotion, but for the majority of research ethics applications, an ethics of space requires room for deliberation and fluidity in the assessment of risks, benefits, and social and scientific value. Little change would need to occur in the extant regulatory framework to acknowledge the importance of 'tolerances' (as opposed to bright lines or thresholds) in REC deliberation regarding whether participants are adequately protected and the ways in which research can be improved. Regulations could be more explicit in delineating the functions of RECs to protect and promote. While the GAfREC suggest that RECs have a primary role of participant protection and a secondary role of promoting the interests of research, researchers, and the public, a clearer charting of functions-treating these not as primary and secondary per se, but rather as relational values that are deliberated in a fluid manner-would likely improve inter-regulator relations as well as researchers' (and publics') understanding of what RECs do. RECs, it is suggested, have the twin role of participant protection and research promotion, but they also have an educational role in increasing knowledge and awareness of ethical issues and regulations; an advisory role in guiding researchers, sponsors, and institutions; as well as a conciliatory role in helping adjudicate potential conflicts between researchers and participants. ${ }^{26}$

Feedback loops can be further developed in the regulatory framework. These are closely connected to 'regulatory conversations' as discussed below. A processual-oriented mode of regulation (1) recognizes the inherent flexibility and fluidity (and indeed uncertainty) in health research, (2) enables adaptive responses to changes in law and regulation, and (3) helps guide actors through the research process. ${ }^{27}$ Currently, there is weak association between rendering an ethics opinion and learning about its outcome. As I have indicated already, 'ethical research' is not a static concept; feedback loops in the form of electronic communication and face-to-face meetings should be strengthened to encourage RECs to

26 See HSE Research Ethics Committees Review Group, Review of Research Ethics Committees \& Processes in Republic of Ireland (Health Service Executive 2008) 7 .

27 Taylor-Alexander and others (n 20) 158. 
engage in dialogue with researchers, sponsors, and others to continually ensure research is ethical. That is, they can help sustain an environment where participants are adequately protected and research optimizes social and scientific value. Mechanisms also should be developed to foster feedback loops where researchers can re-engage in discussions with RECs and the HRA so as to adapt regulatory processes-leading to ongoing improvement and an evidence-based framework. This would help ensure regulatory processes are effective and cost-justified, and also increase expertise in decision-making. A more evidence-based framework would not only enable REC members to improve their ability to make good decisions, it would also make the process more transparent and enable (managing) regulators and publics alike to evaluate the effectiveness of REC decisions in protecting participants and promoting research.

\subsubsection{Enhanced regulatory connectivity}

We saw in Chapter 5 that regulations such as the GAfREC are ambiguous in delineating the relationship of science and law to ethics review, and fail to capture the inherent connections between these regulatory spaces. A relatively minor amendment to the regulatory framework would be to revise the GAfREC and other regulations to account for regulatory connectivity that occurs in practice. The REC's opinion is not a legal opinion, but it is necessarily informed by the law. Likewise, an ethics opinion cannot be achieved without an adequate investigation of and satisfaction with the science. Regulations also should not encourage delegation to other regulatory bodies out of concern for potential overlap; such overlaps tend to occur inevitably. Rather, regulations should encourage greater synergy, not to mention greater efficiencies, among RECs and other bodies such as the MHRA, data monitoring committees, and data access committees.

\subsection{Conversations}

To foster regulatory responsiveness, RECs should be encouraged to engage in discussions and negotiations with researchers, sponsors, and other actors before an application is submitted to the REC, as well as after a proposal has received a favourable opinion. These conversations may revolve around ethical concerns that have arisen during the course of the project, but they may also go beyond this. Figure 6.1 represents where opportunities arise for RECs and other actors to engage in 


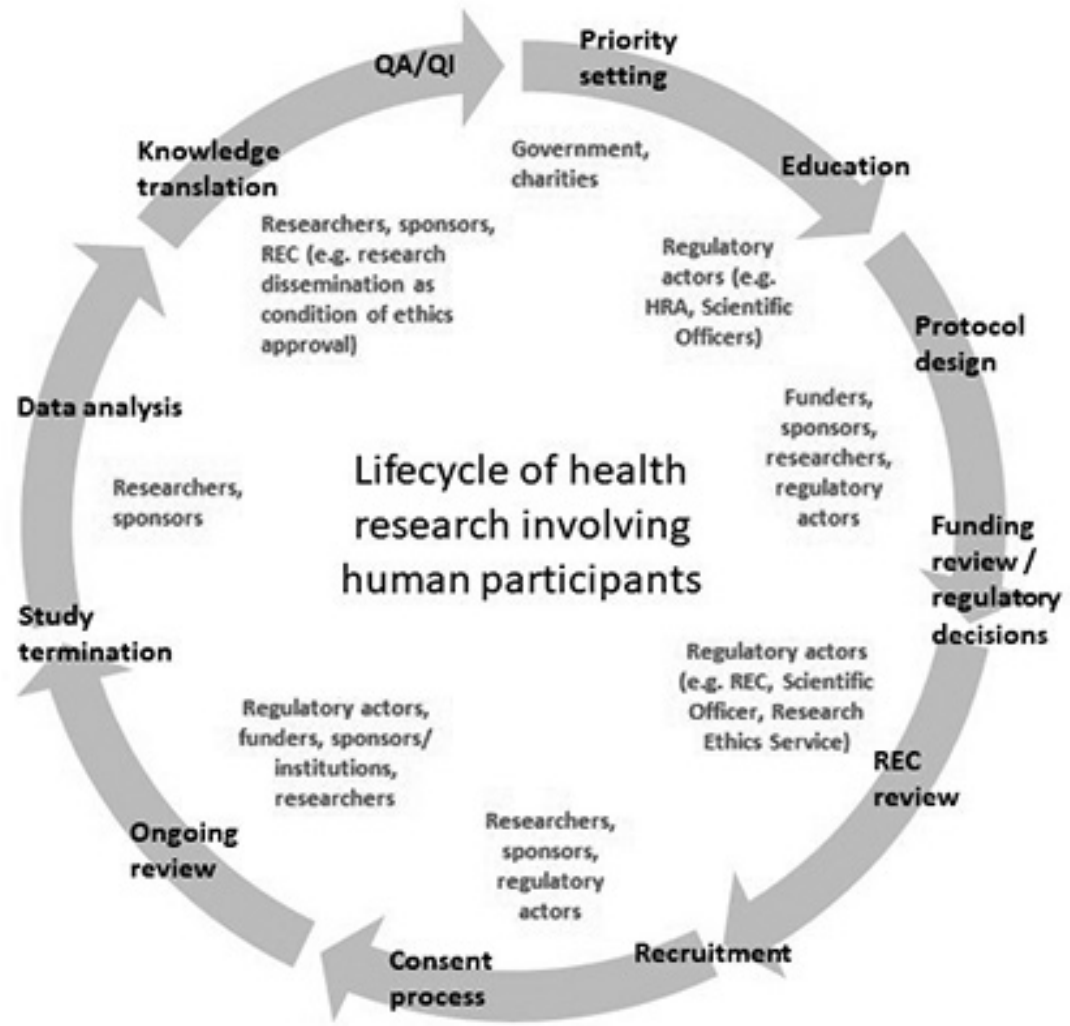

Note: $\quad \mathrm{QA} / \mathrm{QI}=$ quality assurance/quality improvement

Figure 6.1 Lifecycle of health research involving human participants, with proposed augmented roles for actors to engage in 'regulatory conversations' across different elements of the lifecycle ${ }^{28}$

'regulatory conversations' 29 with researchers, sponsors, and institutions across the lifecycle.

James Anderson and colleagues identify 12 elements in the lifecycle of health research involving human participants: (1) priority setting; (2) education-scientific and ethical; (3) protocol design; (4) funding

28 Figure adapted from James Anderson and others, 'Research Ethics Broadly Writ: Beyond REB Review’ (2011) 19 Health Law Review 12.

29 See Black (n 10). 
review; (5) ethics review; (6) recruitment; (7) informed consent; (8) monitoring; (9) project termination; (10) data analysis; (11) knowledge transfer; and (12) quality assurance (QA) and quality improvement (QI). ${ }^{30}$ At first glance, it would seem that RECs address only a narrow portion of issues within the lifecycle. Yet the evidence from the empirical research suggests that RECs, as health research regulators, in fact address many other (but certainly not all) elements in the lifecycle.

A revamped regulatory framework for regulatory stewardship should enable managing regulators, RECs (and particularly REC Chairs), and actors such as (or similar to) Scientific Officers to support researchers and sponsors in working through other elements in the lifecycle, including ethical and regulatory education (i.e. how to work through the ethics review process), protocol design, and issues concerning recruitment, consent, ongoing review, and knowledge transfer (e.g. communication of results). This is not to suggest these regulatory actors must necessarily play a substantive role in these other elements of the lifecycle (and indeed such a normative position would require consideration of resources and infrastructure). Rather, it is to suggest that these regulatory actors should be encouraged to further engage others and each other in these additional elements - reflecting to a large degree what they already do in practice-with a view towards promoting socially valuable and ethical research. Likewise, it is to suggest that researchers and sponsors should be strongly encouraged to engage in regulatory conversations with their regulators, before, during, and after the launch of a research project.

\subsubsection{Sounding board and discourse ethics}

We saw in Chapters 2 and 3 that a common past criticism of RECs has been that they engage in a 'tick-box' bureaucratic ethics rather than a deliberative ethics. If research ethics is to be seen as more than rigid application of rules and standards, it must be allowed to flourish through discourse. ${ }^{31}$ Linus Johnsson and colleagues argue that ethics review should be 'an arena for researchers to discuss their research, receive advice, and practise their ethics skills, and guidelines to be generally applicable, value-based and inspirational rather than specific, rule-based and regulative' 32 (if we take the term 'regulative' to mean controlling and

\footnotetext{
30 ibid 13-14.

31 This argument is advanced more fully in David Townend and Edward Dove, 'Approaching Ethics Review Equivalency Through Natural Justice and a "Sounding Board” Model for Research Ethics Committees' (2017) 36 Medicine and Law 61.

32 Johnsson and others (n 23) 43.
} 
compliance-driven). Regulatory actors such as the HRA, RECs, and Scientific Officers should be encouraged to engage in informal dialogue with researchers (as well as institutions and sponsors) to offer them guidance through regulatory pathways. ${ }^{33}$ Similarly, researchers and sponsors should be encouraged to speak with regulatory actors to provide them with on-the-ground information regarding a research project: how it is developing, whether any roadblocks or surprises have emerged, or whether there has been any deviation between the approved protocol and the actual conduct of the research.

In-person REC meeting attendance by researchers should continue to be strongly recommended, but not required. Researchers should be made aware that deliberations can be unpredictable (a REC that favourably approves an application upon internal deliberation will not need to then speak with the researcher, and thus a researcher may risk 'wasting' resources in attending). A face-to-face meeting will not guarantee a certain outcome, but it may increase the chance that a REC will render a provisional opinion as opposed to an unfavourable opinion. ${ }^{34}$ Thus, a recommendation for face-to-face meetings should clarify the benefits that may accrue: not only a decreased risk of an unfavourable opinion, but an opportunity to engage with a REC to protect participants and promote ethical research through a dynamic, nuanced ethical discourse. Ideally, this encounter should be in person, but if not, managing regulators should ensure there are proper resources for RECs to engage in reliable telecommunication (e.g. video or teleconference) with researchers.

\subsection{Stewardship}

Regulatory stewardship involves different actors helping researchers, sponsors, and institutions navigate complex regulatory pathways and work through the thresholds of regulatory approvals. Collective responsibility also defines regulatory stewardship. In the case of health research, collective responsibility involves regulators and regulatees alike working together to design and conduct research that is ethical and socially and scientifically valuable, and that ultimately aims to improve human health. This can only be accomplished if regulators and regulatees communicate

33 Robert Klitzman refers to this as 'curbside consults' with researchers. See Robert Klitzman, The Ethics Police? The Struggle to Make Human Research Safe (OUP 2015) 330-31.

34 Peter Heasman and others, 'Does Researchers' Attendance at Meetings Affect the Initial Opinions of Research Ethics Committees?' (2008) 4 Research Ethics Review 56. 
with one another and make clear who has what responsibility and role to be played (if any) at each stage in the research lifecycle.

To be clear, then, regulatory stewardship involves different actors serving not in a protecting capacity alone, but also in a capacity to promote the pursuit of clearly identified ends, including ethically robust, scientifically sound research. In so doing, stewards can help reduce regulatory burdens and achieve proportionality in research ethics review and oversight. While this function is performed currently by different non-REC actors relatively well (e.g. NHS R\&D Forum, ${ }^{35}$ MRC Regulatory Support Centre, ${ }^{36}$ institutions that may create regulatory knowledge and support programmes to support their researchers ${ }^{37}$ ), more stewardship support can and should be provided by RECs and managing regulators such as the HRA. Indeed, a key feature of regulatory stewardship is that it may be practised as much by non-state actors as by state actors charged with formally prescribing and proscribing actions under the law. Here, opportunities are present for several regulatory actors to assist researchers, sponsors, and others in manoeuvring complex regulatory regimes. For example, we have seen that the four Scientific Officers in Scotland provide an immense amount of support to RECs, researchers, and sponsors alike. There is no Scientific Officer equivalent in the other three nations. Here, the HRA may consider creating equivalent positions in England. To do so, it may not need to create multiple Scientific Officers in each of the HRA's Regional Offices. Instead, it can revive its effort to create a REC Application Review and Advice Service staffed by independent experts who may have had previous experience in chairing or managing RECs, as well as experience in health research and regulation.

Regulation should more clearly provide channels for RECs and managing regulators to engage with researchers and sponsors in improving the quality of research protocols and applications and in working through law, regulation, and regulatory approvals. These channels could include enhanced online toolkits provided by the HRA coupled with online support or through meetings in HRA Regional Offices.

35 NHS Research \& Development Forum <www.rdforum.nhs.uk/content/> accessed 22 October 2019.

36 MRC Regulatory Support Centre <www.mrc.ac.uk/research/facilities-andresources-for-researchers/regulatory-support-centre/> accessed 22 October 2019.

37 See e.g. University of Edinburgh, 'Academic and Clinical Central Office for Research and Development' <www.accord.ed.ac.uk/> accessed 22 October 2019. 
Regulatory stewardship also could be put on a legal basis. For example, in New Zealand, one of the purposes of the State Sector Act 1988, as amended, 'is to promote and uphold a State sector system that [...] fosters a culture of stewardship'. ${ }^{38}$ The Act defines 'stewardship' as the 'active planning and management of medium- and long-term interests, along with associated advice'. ${ }^{39}$ The Care Act 2014 goes to some length to enact stewardship by confirming as a matter of law that health research regulatory agencies have responsibilities not just to protect research participants' interests, but also to promote ethical and safe research. Yet further legal footing can be provided by declaring through statutory regulation, guidance, or otherwise that health research regulatory agencies are expected to bring a more systematic, comprehensive, lifecycle approach to the management of existing regulation, which in this context, would mean ensuring that regulations are: (1) proportionate; (2) fit for purpose; (3) enabling for stewards to work with researchers and others in achieving their desired ends; and (4) enabling for regulators to articulate how the public interest will be promoted through research. Such a legal footing would clarify the value of different actors in enacting regulatory stewardship across the research lifecycle, and also avoid constricting the roles and procedural and substantive aspects of actors in rigid law that can be counterproductive to the value of flexibility that is inherent in liminality.

Stewardship is a heterogeneous concept. Given the various actors who can serve in a stewardship capacity, regulation should be designed to promote specific (but not necessarily narrow) tasks for different actors. Depending on the context, these different actors could be labelled as state stewards (e.g. the Department of Health and Social Care and the HRA must act in a manner deemed to contribute to the public interest, for example as established by law), operational stewards (e.g. REC Managers or Scientific Officers who help usher researchers through the complexity of established procedures such as ethics application processes), and ethics stewards (e.g. RECs that act to protect participants and promote research). At the same time, as I and colleagues have argued elsewhere:

It would follow also from this that researchers must be trained in, and made aware of, this central role in making (good) research happen. As a minimum, this would require researchers to acknowledge their role in contributing to

\footnotetext{
38 State Sector Act 1988, as amended 2013 [NZ], s 1A.

39 ibid s 32.
} 
streamlined regulation by responsible discharge of duties to work with regulators effectively. ${ }^{40}$

By stating clearly what roles each actor should play at the different stages in the research lifecycle, and how each actor should work with others to move from one stage to the next (i.e. how and when 'the mantle should be passed'), health research regulation could achieve more robustly the twin aims of participant protection and research promotion.

\section{CONCLUSION}

In this chapter, I further unpacked the significance of the liminality of RECs and the ability of actors within the health research regulatory space to serve as regulatory stewards. I did so by charting how protection and promotion can and should work together, and by suggesting a normative model of what a new regulatory framework for health research oversight ought to look like if it were to explicitly endorse regulatory flexibility, conversations, and stewardship. I suggested that an iterative view of protection and promotion defined by tolerance for fluidity would better recognize the liminal and thus processual enterprise of health research. I also argued that regulation would be well served if it accounted for the roles that RECs and other actors (such as Scientific Officers) can play across the lifecycle of research by engaging in 'conversations' with researchers and sponsors (among other actors, such as funders). Providing such a space for flexibility and experimentation across the research lifecycle would allow for greater opportunities for 'regulatory play' to emerge, and in so doing foster an environment that both protects research participants and also facilitates responsible health research through proportionate regulation and coordinated alignment of ethics review and other regulatory processes.

In the concluding Chapter 7, I recap the arguments of this book, my main research findings, and possible future directions for research.

\footnotetext{
40 Laurie and others (n 21).
} 